\title{
SYSTEM MATRIX COMPUTATION FOR ITERATIVE RECONSTRUCTION ALGORITHMS IN SPECT BASED ON DIRECT MEASUREMENTS
}

\author{
DAMIAN BORYS ${ }^{*, * *}$, KATARZYNA SZCZUCKA-BORYS **, KAMIL GORCZEWSKI ** \\ * Institute of Automatic Control \\ Silesian University of Technology, ul. Akademicka 16, 44-100 Gliwice, Poland \\ e-mail: damian.borys@polsl.pl \\ ** Nuclear Medicine and Endocrine Oncology Department \\ Comprehensive Cancer Centre, Maria Skłodowska-Curie Memorial Institute \\ ul. Wybrzeże Armii Krajowej 15, 44-101 Gliwice, Poland \\ e-mail: kgorczewski@io.gliwice.pl
}

\begin{abstract}
A method for system matrix calculation in the case of iterative reconstruction algorithms in SPECT was implemented and tested. Due to a complex mathematical description of the geometry of the detector set-up, we developed a method for system matrix computation that is based on direct measurements of the detector response. In this approach, the influence of the acquisition equipment on the image formation is measured directly. The objective was to obtain the best quality of reconstructed images with respect to specified measures. This is indispensable in order to be able to perform reliable quantitative analysis of SPECT images. It is also especially important in non-hybrid gamma cameras, where not all physical processes that disturb image acquisition can be easily corrected. Two experiments with an ${ }^{131} I$ point source placed at different distances from the detector plane were performed allowing the detector response to be acquired as a function of the point source distance. An analytical Gaussian function was fitted to the acquired data in both the one- and the two-dimensional case. A cylindrical phantom filled with a water solution of ${ }^{131} I$ containing a region of "cold" spheres as well as a uniform solution (without any spheres) was used to perform algorithm evaluation. The reconstructed images obtained by using four different of methods system matrix computation were compared with those achieved using reconstruction software implemented in the gamma camera. The contrast of the spheres and uniformity were compared for each reconstruction result and also with the ranges of those values formulated by the AAPM (American Association of Physicists in Medicine). The results show that the implementation of the OSEM (Ordered Subsets Expectation Maximization) algorithm with a one-dimensional fit to the Gaussian CDR (Collimator-Detector Response) function provides the best results in terms of adopted measures. However, the fit of the two-dimensional function also gives satisfactory results. Furthermore, the CDR function has the potential to be applied to a fully 3D OSEM implementation. The lack of the CDR in system matrix calculation results in a very noisy image that cannot be used for diagnostic purposes.
\end{abstract}

Keywords: SPECT, iterative reconstruction, quantitative imaging.

\section{Introduction}

Nuclear medicine imaging can be used to generate a spatial or temporal activity distribution of a radioisotope administered to the subject of a study. Like in many other modalities, the $3 \mathrm{D}$ distribution is determined from $2 \mathrm{D}$ representations of the 3D distribution. The activity distribution of interest is projected onto a flat detector. Gamma rays which are not perpendicular to the surface of the detector are filtered out by a parallel-hole collimator. Other collimator types such as a pin-hole or a fan-beam are not within the scope of this paper. The detector rotates around the centre of rotation acquiring a projection for each angle. The matrix containing projection measurements for all angles is referred to as a sinogram and is used in the reconstruction process.

Reconstruction algorithms proposed in the literature (Cherry et al., 2003; Zeng, 2001; Zaidi, 2006) can be divided into two classes: analytical and iterative. The former assume noiseless data, an ideal collimator, no attenuation and no scatter of gamma radiation. These assumptions can make the reconstruction result an inaccurate representation of the true activity distribution. Therefore, additio- 
nal data filtering and postprocessing are necessary. The most commonly used method is the Filtered Back Projection (FBP) algorithm (Zaidi, 2006), based on the inverse Radon transform. The FBP method introduces streak artifacts when large differences in activity are imaged. The latter (Vandenberghe et al., 2001) allow a complex model of gamma radiation interaction to be taken into account, and statistical variability of measured data. These are recommended for quantitative image analysis (Zaidi, 2006) which is crucial, for example, in dosimetry. In the commercial software provided with the gamma camera system the possibility to adjust various parameters is limited. It is especially important in non-hybrid systems, where possible corrections are restricted to those based only on the emission data.

The aim of our work was to obtain the best quality reconstructed images in terms of specified measures. It is paramount to perform reliable quantitative analysis of SPECT images, especially in non-hybrid gamma cameras as the necessary corrections are more challenging than in SPECT/CT cameras.

The OSEM algorithm was implemented to allow the implementation of all reconstruction corrections since this is not possible with the software provided by the gamma camera manufacturer. A set of phantom measurements was performed to obtain parameters necessary to compute the system matrix. The approach developed herein is based on direct measurements. Finally, phantom tests were performed to verify which method was most useful according to the specified measures.

\section{Image reconstruction problem formulation}

In clinical routine the reconstruction of 3D data is usually performed as a set of $2 \mathrm{D}$ disjoint reconstruction problems that result in a set of transaxial slices. The surface of the detector is pixelated; therefore, the discrete description of the image space is widely used. However, with this approach the $3 \mathrm{D}$ nature of photon interaction is neglected and an assumption is needed to limit all interactions to the thickness of one slice (voxel size).

An unknown activity distribution of interest can be represented as an $N \times N \lambda$-matrix, where $N$ is typically 128 (maximum 256 because of the time needed to acquire a useful number of counts). For ease of numerical computations, $\lambda$ is often reshaped into a $1 \times B$ vector, where $B=N^{2}$. A sinogram $n$ is an $A \times N$ matrix, where $A$ enumerates the angles. For the same reasons as in the case of $\lambda$, the sinogram $n$ is reshaped to a $1 \times A N$ vector.

A system matrix $(\mathbf{p})$ connects the unknown activity distribution vector $\lambda$ with the measurement vector $n$ :

$$
n=\mathbf{p} \cdot \lambda \text {. }
$$

It is an $A N \times N^{2}$ matrix. The physical meaning of its element $p_{i j}$ is the probability that the element $\lambda_{i}$ produces a signal in a given detector element at a certain angle $\left(n_{j}\right)$. The size of the matrix $\mathbf{p}$, assuming projections of $128 \times$ 128 pixels acquired from 64 angles, is $64 \times 128^{3}$, which is over $134 \times 10^{6}$ elements. Since a very sparse and largescale matrix $\mathbf{p}$ is very difficult to invert, which would be the straightforward way to compute the unknown $\lambda$ from the measured signal $n$, it is necessary to use an iterative approach to retrieve the activity distribution of interest.

In nuclear medicine the acquired count values are rather low, so the data tend to be noisy. Therefore, statistical reconstruction methods seem to be a reasonable choice, although we must remember that it is impossible to find a true solution from such data. Many assumptions about the noise can be made, but for the emission data the Poisson model seems to be most adequate.

One of the most commonly used statistical iterative algorithms is the Maximum Likelihood Expectation Maximization (MLEM) algorithm (Shepp and Vardi, 1982) and its modification - the Ordered Subsets Expectation Maximization (OSEM) algorithm (Hudson and Larkin, 1994).

2.1. Maximum likelihood expectation maximization algorithm. Let $n^{*}(d)$ be the measurement in the detector element $d$, for $d=1,2 \ldots, D$. The measured data can be described by a Poisson distribution and have the probability mass function given by

$$
f\left(n^{*} \mid \lambda^{*}(d)\right)=e^{-\lambda^{*}(d)} \frac{\lambda^{*}(d)^{n^{*}(d)}}{n^{*}(d) !},
$$

where

$$
\lambda^{*}(d)=\sum_{b=1}^{B} \lambda(b) p(b, d)
$$

is the expected value of $n^{*}(d)$, and $\lambda(b)$ represents the unknown activity distribution at position $b$. The maximum likelihood estimator $L(\lambda)$ is connected with the measured data and can be expressed as

$$
L(\lambda)=\prod_{d=1, \ldots, D} e^{-\lambda^{*}(d)} \frac{\lambda^{*}(d)^{n^{*}(d)}}{n^{*}(d) !}
$$

The logarithm of the function $L(\lambda)$ can be used instead to simplify the calculation, because this operation does not change the maximum of the function. If we write $l(\lambda)=\log L(\lambda)$, the expression becomes

$$
l(\lambda)=\sum_{d=1}^{D}\left[-\lambda^{*}(d)+n^{*}(d) \log \lambda^{*}(d)-\log n^{*}(d) !\right] .
$$

The formula of the final algorithm can by obtained by finding a local maximum using the derivative of Eqn. (5) 
with respect to $\lambda(b)$ :

$$
\lambda^{(k+1)}(b)=\frac{\lambda^{(k)}(b)}{\sum_{d=1}^{D} p(b, d)} \sum_{d=1}^{D} \frac{n^{*}(d) p(b, d)}{\sum_{b^{\prime}=1}^{B} \lambda^{(k)}\left(b^{\prime}\right) p\left(b^{\prime}, d\right)} .
$$

\subsection{Ordered subsets expectation maximization algo-} rithm. Many practical implementations of the algorithm shown by Eqn. (6) were described by Zaidi et al. (2006). Most of them focused on reconstruction quality, omitting the problem of computation time. Hudson and Larkin (1994) modified the MLEM algorithm in a way that allowed a significant reduction in the evaluation time and made practical applications possible. In the proposed idea, the measurements were divided into independent subsets, and then the maximum of Eqn. (6) was found using successive subsets of the measurements in successive iterations. In this way, the achieved acceleration in computation time was almost equal to the number of subsets.

This method is referred to as the OSEM algorithm and can formally be written as

$$
\begin{array}{r}
\lambda^{(k+1)}(b) \\
=\frac{\lambda^{(k)}(b)}{\sum_{d \in J m}^{S} p(b, d)} \sum_{d \in J m}^{S} \frac{n^{*}(d) p(b, d)}{\sum_{b^{\prime}=1}^{B} \lambda^{(k)}\left(b^{\prime}\right) p\left(b^{\prime}, d\right)},
\end{array}
$$

where $S$ is the number of independent subsets: $J_{1}, J_{2}, \ldots, J_{S}$.

Any strategy can be used to assign measured data to a specific subset. However, the choice of the strategy has an effect on the convergence quality and speed of the algorithm. The most commonly used strategy (Bruyant, 2002) is to successively assign measurements to subsets.

2.3. System matrix p. The crucial factor, beside the quality of the acquired images, is the system matrix ( $\mathbf{p}$ matrix). Each element $p(b, d)$ gives the probability of detecting the photon emitted from the image point $b$ in the detector at point $d$. The basis of the system matrix construction is gamma camera geometry. It depends on the field of view of the detector point (Fig. 1). Assuming ideal collimation of the beam, this matrix can be determined using a ray-based or a pixel-based approach (Bruyant, 2002). In the easiest case we can consider that an ideal collimator has an infinite length and totally nonpenetrable septa resulting in the situation where the detector point's observable area is very narrow and formed by two parallel lines (Fig. 1, solid lines). If the finite length of the collimator's hole (for high energy collimators it is $5.08 \mathrm{~cm}$ ) is taken into account, it is necessary to extend the field of view of the detector point (Fig. 1 dotted line). The second case was described analytically by Loudos (2008)

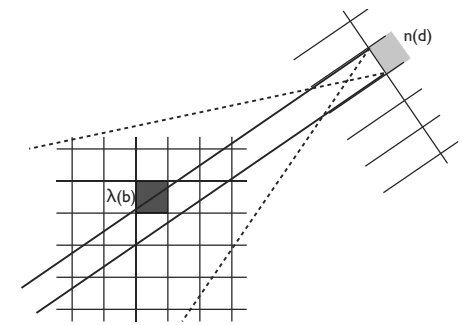

Fig. 1. System matrix $\mathbf{p}$ whose element $p(b, d)$ gives the probability of detecting the photon emitted from the image point $b$ at the detector point $d$. The field of view is marked with solid lines for an ideal collimator and with dotted lines when the finite size of the collimator is taken into account.

including the hexagonal shape of collimator holes, and it was used to compute the system matrix.

The detector response also depends on the interaction of the gamma rays with the collimator septa so the detector counts photons that were scattered in the collimator septas or passed through them as well as those photons that go through the collimator holes. Autret et al. (2005) showed the impact of the scattered and septal penetration photons in the projection images by means of Monte Carlo simulations.

An alternative to the analytical methods mentioned is to compute the system matrix using Monte Carlo simulations (Rafecas et al., 2004; Lazaro et al., 2005). The accuracy of this method depends on the models used in the simulation. However, in this case, a full model of the measurement equipment can be included in the system matrix. Some papers (Lazaro et al., 2005) show very interesting results but also indicate that, because of computational complexity, the use of MC methods in clinical routine can nowadays be problematic.

The method presented here is based on direct measurement of the detector response to a point source. It is the most straightforward way to compute a system matrix, especially when only the interpolation of the measurements is used. An analytical expression could be found with appropriate parameters because the data suggest a Gaussian shape to the response function. In this way, by knowing the distance between the image point from the detector plane and the detector centre, the p-matrix elements can be computed.

There are a few similar solutions in the literature that also treat the problem of spatial system response compensation. Formiconi et al. (1989) used a line source to obtain the FWHM of the Gaussian response function. In the work of Liang et al. (1992), a linear dependence of the FWHM parameter on the distance between the point source and the detector was also found. A Gaussian function was also fitted to point source measurements by Gilland 
et al. (1994). In all the works where the detector response was included in the reconstruction process the authors declared improved resolution and noise characteristics of the reconstructed images. In the present paper, point source measurements were carried out which are easier to perform than in the line source case. Two dimensional projections were used to obtain the appropriate detector response function and to validate its usefulness in reconstruction. The higher resolution of the measurements was also examined in relation to the quality of the detector response function fit.

\section{Materials and methods}

3.1. Gamma camera response measurements. In this work, a Siemens e.Cam Duet gamma camera was used, equipped with a 1 inch thick $\mathrm{NaI}(\mathrm{Tl})$ crystal. A High Energy (designed for ${ }^{131} I$ study), parallel, hexagonal shaped hole collimator was used with an effective spatial resolution of $22.3 \mathrm{~mm}$. Other characteristics of the collimator include $8 \times 10^{3}$ holes, hole length: $50.8 \mathrm{~mm}$, septal thickness: $2 \mathrm{~mm}$, hole diameter: $3.4 \mathrm{~mm}, 3.5 \%$ septal penetration.

The focus of this work was the ${ }^{131} I$ isotope which has its main photopeak energy at $364 \mathrm{keV}$, physical halflife of 8.02 days and is used to diagnose and treat many thyroid diseases, including thyroid cancer.

Two experiments were conducted. In the first one, an ${ }^{131} I$ point source of $1 \mathrm{MBq}$ activity was measured with one detector at distances ranging from 5 to $29 \mathrm{~cm}$ counting from the gamma camera head in $2 \mathrm{~cm}$ steps. The matrix size was set to $128 \times 128$ points and the zoom was set to 1 with the resulting pixel size of $4.795 \times 4.795 \mathrm{~mm}^{2}$. The acquisition time was adjusted with the source-gamma camera distance to obtain a constant count of $3 \times 10^{4}$.

In the second experiment, an ${ }^{131} I$ point source of 13 $\mathrm{MBq}$ of the activity was used to measure the detector response in the distance range from 7 to $31 \mathrm{~cm}$ in $2 \mathrm{~cm}$ steps using two detectors at the same time ( 7 to $17 \mathrm{~cm}$ for Detector 1 and 21 to $31 \mathrm{~cm}$ for Detector 2). The matrix size was set to a maximum available value of $1024 \times 1024$ points and the zoom was set to 1 with the resulting pixel size of $0.599 \times 0.599 \mathrm{~mm}^{2}$. The acquisition time was constant and set to 20 minutes for each distance.

The second experiment with the use of the highest available resolution was performed in order to obtain better function adjustment and better parameter fit than in the standard resolution.

The final verification of the implemented OSEM reconstruction algorithm was conducted with a point source without attenuation material, with 8 iterations and 4 subsets in each case.

3.2. Collimator-detector function. For every measurement in the first experiment, a profile at a maximum va- lue was used to find the analytical function. Considering the shape of the plotted profiles and after a few tests with different fitting functions, it was decided that the Gaussian function was satisfactory. Then, the analytical function, which was described by

$$
f_{d}(x, a, b, c)=a \exp \left(\frac{-(x-b)^{2}}{c^{2}}\right),
$$

was fitted to the obtained data, where the parameters $a, b$, $c$ are distance dependent. Functions describing those parameters in relation to the distance were found as described in Section 4.

In the second experiment, a surface fitting was performed. The analytical function was described by

$$
\begin{aligned}
& f_{d}\left(x, a, b_{x}, b_{y}, c_{x}, c_{y}\right) \\
& =a \exp \left(-\frac{\left(x-b_{x}\right)^{2}}{c_{x}^{2}}-\frac{\left(y-b_{y}\right)^{2}}{c_{y}^{2}}\right) .
\end{aligned}
$$

This case allows the 3D nature of the photon interactions that occur in the detector plane to be taken into account. In later computations, however, we applied this fitting to a standard reconstruction algorithm performed in a sliceby-slice way.

3.3. OSEM verification. To verify the OSEM algorithm and $\mathbf{p}$ matrix computations, phantom measurements were performed with a $20 \mathrm{~cm}$ high plexiglass cylinder filled with a ${ }^{131} I$ water solution of $500 \mathrm{MBq}$ activity. Inside the phantom there were 6 "cold" spheres (filled with water, without activity). The phantom is presented in Fig. 2. The spheres diameters were $3.85,3.0,2.4,1.85,1.5,1.15$ $\mathrm{cm}$.
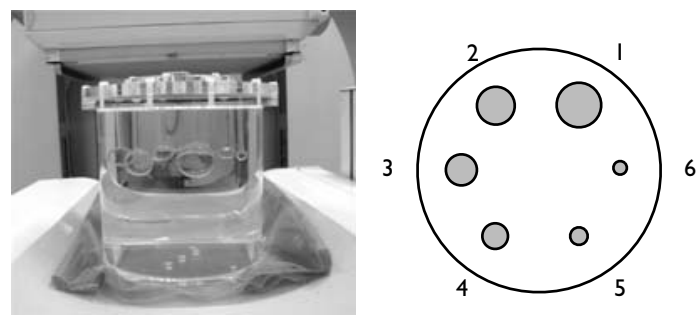

Fig. 2. Phantom for the validation and verification of the reconstruction algorithm. The phantom was filled with a ${ }^{131} I$ water solution and 6 "cold" spheres were placed inside. General view of the phantom (left) and schematic representation of the "cold" spheres.

3.3.1. Computation of the matrix p. In this work the matrix $\mathbf{p}$ was determined in four ways:

- including a basic point-detector geometry function as shown in Fig. 1 (solid lines), $p(b, d)=a(b, d)$; 
- including an intrinsic crystal response and geometric, septal penetration and septal scatter components that depend on the collimator characteristics (Collimator Detector Response-CDR) based on the first measurement and interpolation, $p(b, d)=p_{\mathrm{CDR}_{\text {interp }}}(b, d)$;

- including a CDR function obtained from the set of fitting functions for the first experiment, $p(b, d)=$ $p_{\mathrm{CDR}_{1 \mathrm{D}}}(b, d)$;

- including a CDR function obtained from the surface fit of the second experiment data, $p(b, d)=$ $p_{\mathrm{CDR}_{2 \mathrm{D}}}(b, d)$.

3.3.2. Reconstruction tests. In all cases a photon attenuation was included based on the attenuation map derived from the CT scan registered with the SPECT study (Borys et al., 2006). The CT scan provides an electron density map of the phantom in Hounsfield Units. These allow a linear attenuation coefficients distribution to be obtained. These coefficients were used to calculate an attenuation map. In the case of the presented phantom, one attenuation coefficient $\left(\mu=0.11\left[\mathrm{~cm}^{-1}\right]\right.$ for ${ }^{131} I$ in water, (Hubbell and Seltzer, n.d.)) was used for the whole phantom as it was filled with the water solution resulting in a uniform density of the examined volume. All data were scatter corrected with the TEW (Triple Energy Window) method (Ichihara et al., 1993), and the dead time effect was removed.

Five reconstructions were made with different $\mathbf{p}$ matrices using measurements of the phantom:

- Reconstruction A: system matrix based only on ideal collimator geometry $a(b, d)$;

- Reconstruction B: system matrix based on the CDR from the interpolation;

- Reconstruction C: system matrix based on the CDR from the 1D fitting function;

- Reconstruction D: system matrix based on the CDR from the 2D fitting function;

- Reconstruction E: result obtained with software included with the gamma camera.

Two slices from each reconstruction were analyzed: the one with the cold spheres and then that which was considered a uniform part of the phantom. The quality of the reconstruction was estimated by the contrast of the "cold" spheres (Eqn. (10)) and by the integral uniformity (Eqn. (11)), as well as the root-mean-square noise (RMS, Eqn. (12) ) (Graham et al., 1995) for the uniform region of the phantom. In addition to the measures used by Graham et al. (1995), a differential uniformity was added. It is expressed by the same equation as for the integral uniformity (Eqn. (11)), but the integral uniformity is determined for a small $(15 \times 15$ pixels $)$ square central Region Of Interest (ROI) and differential uniformity is determined for every point of this central ROI including a 5-point neighbourhood. It is calculated consecutively for the vertical and horizontal neighbourhood and the maximum value is taken as a result. The following parameters were used for quality evaluation:

$$
\begin{aligned}
C & =\frac{P_{\mathrm{avg}}-P_{\mathrm{min}}}{P_{\mathrm{avg}}}, \\
U_{\text {int }} & =\frac{P_{\mathrm{max}}-P_{\min }}{P_{\max }+P_{\min }}, \\
\mathrm{RMS} & =\frac{P_{\mathrm{std}}}{P_{\mathrm{avg}}} \times 100 .
\end{aligned}
$$

$P$ in the above equations represents the count statistics in the appropriate region. In the case of contrast, the mean count in the small central uniform region is $P_{\text {avg }}$ and the minimal count number in the cold sphere is $P_{\text {min }}$. For the uniform area, the measures of uniformity and the RMS noise, all statistics are determined in a small central region: maximum and minimum count, $P_{\max }$ and $P_{\text {min }}$, standard deviation and mean count value, $P_{\text {std }}$ and $P_{\text {avg }}$, respectively. Additionally, we compared our results with those obtained from the OSEM implementation provided by the gamma camera provider (Siemens eSoft). All reconstruction parameters were identical (8 iterations, 4 subsets). In the gamma camera software, the Chang attenuation correction method was also used (Chang, 1978).

3.3.3. OSEM parameters. The basic parameter in the iterative reconstruction algorithm is the number of iterations and, in the case of OSEM, the number of subsets is also important. In all comparisons of the contrast and uniformity measures described above, 8 iterations with 4 subsets were used. To account for this choice, a reconstruction with the use of one of the previously computed p-matrices (Reconstruction C) was performed. The change in all of the above indices with the change in the number of iterations $(1,2,4,6,8,10,12,16,20$ iterations with 4 subsets) was visualized.

The results were compared with the appropriate ranges defined in the work of Graham et al. (1995). The ranges for the consecutive spheres were determined for the "cold" region: Sphere 1 (0.53;0.73), Sphere $2(0.35 ; 0.56)$, Sphere $3(0.21 ; 0.38)$, Sphere 4 $(0.11 ; 0.27)$. Furthermore, ranges for the integral uniformity $(10.7 ; 18.8)$ and RMS $(3.6 ; 7.2)$ were determined.

\section{Results}

4.1. CDR function measurement and fit. The results of the CDR function measurements performed in the first 
experiment are presented in Fig. 3. The measurements were normalized to make the integral of the CDR function equal to unity along the shift direction for each distance. In the case of the second experiment, where the whole surface was taken into account, normalization was applied so as to make the area under the surface equal to unity.

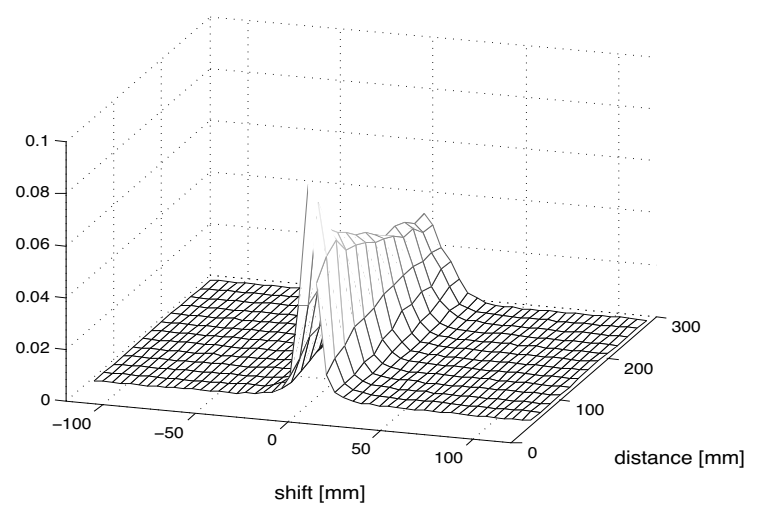

Fig. 3. CDR function as measured for a point source of ${ }^{131} I$ and using the HE collimator. The measurements are normalized for each point source distance.

The measurements were assumed to be Gaussian functions and described by Eqn. (8) and (9). The parameters of this relationship are dependent on the distance between the source and the detector. Therefore, parameters $a, b, c$ were found as a function of distance $(d)$ in order to obtain the best fit of the acquired data. The following parameters and functions minimize the fitting error:

- $a(d)=a_{a} e^{-b_{a} d}$, where $a_{a}=0.124 \pm 0.003$ and $b_{a}=2.77 \pm 0.10 \cdot 10^{-3}$;

- $b(d)=a_{b} d+b_{b}$, where $a_{b}=-5.08 \pm 1.82 \cdot 10^{-3}$ and $b_{b}=0.22 \pm 0.01$

- $c(d)=a_{c} d+b_{c}$, where $a_{c}=0.024 \pm 0.001$ and $b_{c}=3.13 \pm 0.14$.

Two examples of the fitting quality are shown in Fig. 4 for source-detector distances of 5 and $29 \mathrm{~cm}$. The analytical functions fitted to the parameters $a$ and $c$, described above, are shown in Fig. 5

In the second experiment, identical relationships for the CDR function parameters were found, although different parameter values were obtained. The most important parameter values are presented below:

- $a_{a}=0.0055 \pm 0.0005$ and $b_{a}=0.0079 \pm 0.7 \cdot 10^{-3}$;

- $a_{c X}=0.0495 \pm 1.7 \cdot 10^{-3}$ and $b_{c X}=3.7032 \pm 0.35$.

Figure 6 shows the CDR to a point source in the second experiment. The single response of each collimator hole is visible and was the cause of some problems with the Gaussian surface fitting.

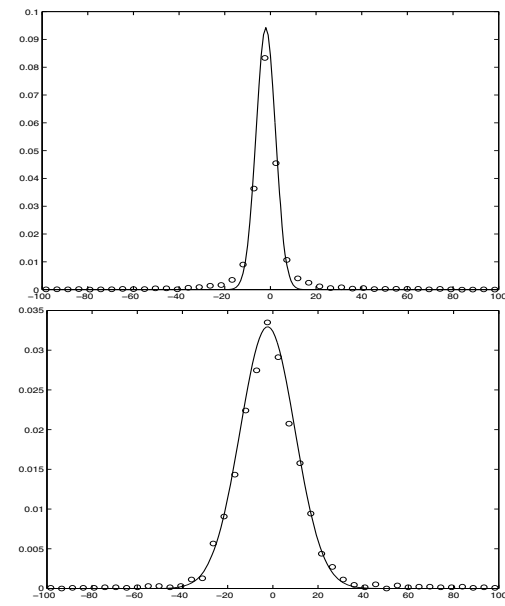

Fig. 4. Example of fitting functions for the first $(5 \mathrm{~cm}$ distance, upper plot) and the last measurement ( $29 \mathrm{~cm}$ distance, lower plot). The measurement points are denoted by circles and the solid line shows the fitted model.
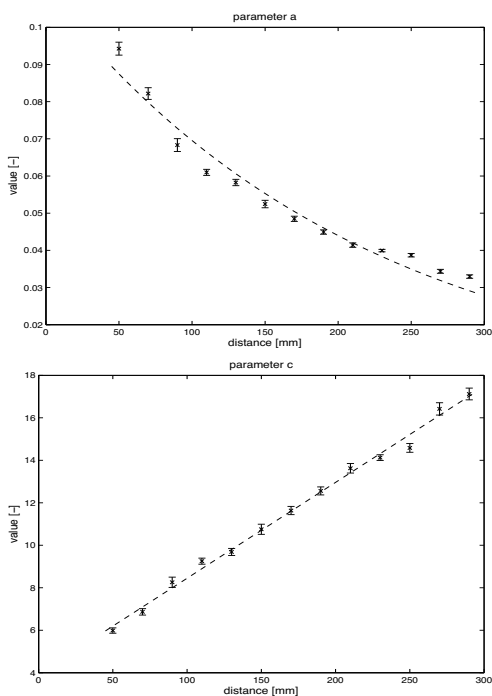

Fig. 5. Analytical functions fitted to the parameters $a$ (upper, exponential plot) and $c$ (lower, linear plot) in the CDR function.

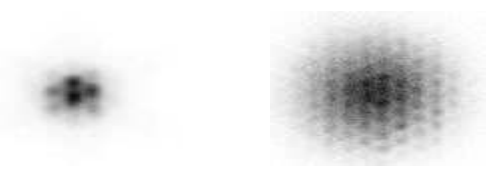

Fig. 6. CDR measurements with the detector matrix set to $1024 \times 1024$ pixels at two distances of the source from the detector: $9 \mathrm{~mm}$ (left plot) and $31 \mathrm{~mm}$ (right plot). 


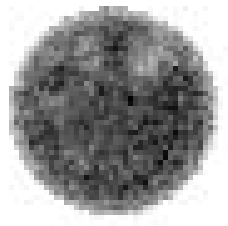

A

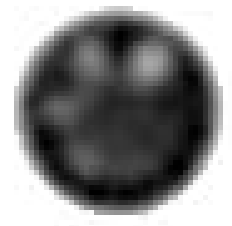

$B$

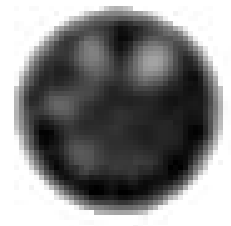

C

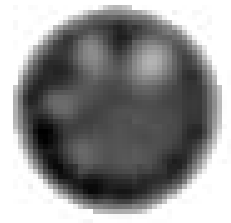

$D$

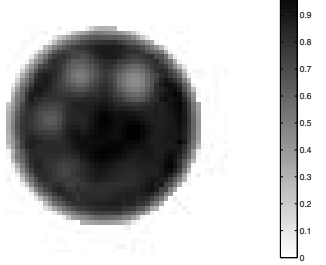

E

Fig. 7. OSEM reconstruction results ( 8 iterations, 4 subsets) for the slice with cold spheres. Letters denote the reconstruction methods applied.

4.2. OSEM verification. The images shown in Figs. 7 and 8 were obtained from the phantom measurement and Reconstructions A-E described above. Visual assessment of the results indicates that only Reconstruction A presents abnormal noise levels. The smallest sphere is not visible on any reconstruction because its diameter was below the effective resolution. Table 1 presents the differences in the contrast of the spheres in the phantom.

The reconstruction of the slice with no spheres is shown in Fig. 8. Since the images were similar, distinguishing reconstruction quality was possible only by comparing the uniformity and RMS parameters. The quantitative parameters describing the uniformity of the reconstructed slice are shown in Table 2. The best (lowest) factors were found for the reconstruction performed by the system provider's software-Reconstruction $\mathrm{E}\left(U_{\text {diff }}\right)$, and for the reconstruction based on the CDR function with interpolation-Reconstruction B $\left(U_{\text {diff }}, R M S\right)$.

The system matrix based on the one dimensional fit of the CDR function (Reconstruction C) gave the highest contrast in the three biggest spheres and other measures that were comparable with the results obtained with other reconstruction methods (for example, integral uniformity).

Reconstruction results B-D present a lower signal level in the centre of the phantom than the standard software result, as shown in Fig. 7 In the slice without the cold spheres (Fig. 8), the centre is more uniform compared with the result of reconstruction E. In this image, ring artifacts are also visible that are not present in the other results.

Table 1. Contrast results for 5 spheres (from $s 1$ to $s 5$ ) for the five different OSEM reconstructions ( 8 iterations, 4 subsets).

\begin{tabular}{|c|c|c|c|c|c|}
\hline recon. & $s 1$ & $s 2$ & $s 3$ & $s 4$ & $s 5$ \\
\hline \hline $\mathrm{A}$ & 0.589 & 0.472 & 0.378 & 0.347 & 0.231 \\
\hline $\mathrm{B}$ & 0.568 & 0.394 & 0.269 & 0.249 & 0.110 \\
\hline $\mathrm{C}$ & 0.570 & 0.414 & 0.286 & 0.229 & 0.081 \\
\hline $\mathrm{D}$ & 0.562 & 0.352 & 0.187 & 0.152 & 0.073 \\
\hline $\mathrm{E}$ & 0.527 & 0.439 & 0.318 & 0.222 & 0.127 \\
\hline
\end{tabular}

Table 2. Uniformity ( $U_{\text {int }}$ : integral and $U_{\text {diff }}$ : differential) and the RMS results for the uniform slice. 5 different OSEM reconstructions ( 8 iterations, 4 subsets).

\begin{tabular}{|c|c|c|c|}
\hline recon. & $U_{\text {int }}$ & $U_{\text {diff }}$ & RMS \\
\hline \hline $\mathrm{A}$ & 42.315 & 42.315 & 13.837 \\
\hline $\mathrm{B}$ & 11.597 & 8.017 & 4.736 \\
\hline $\mathrm{C}$ & 12.145 & 8.240 & 5.001 \\
\hline $\mathrm{D}$ & 15.117 & 9.937 & 6.571 \\
\hline $\mathrm{E}$ & 12.310 & 7.971 & 5.452 \\
\hline
\end{tabular}

The reconstruction algorithm was checked with a point source measurement without any attenuation material using 8 iterations and 4 subsets in each case. The results are shown in Fig. 9

Fig. 9. Point source reconstruction verification: reconstruction with the gamma camera software as a reference (left plot) and the implemented OSEM reconstruction, system matrix built with a $1 \mathrm{D}$ fit CDR function (right plot).

4.2.1. Investigation into the iteration number. The relationship between contrasts of the spheres, the integral and differential uniformity, RMS and the number of iterations were investigated. The results are shown in Figs. 1012. If the range for a value was given in the AAPM report, then it was marked in plots as a solid horizontal line. The presented plots show that the minimal number of iterations is 8 , assuming 4 subsets (see Figs. 10 and 11, plot for Sphere 1 and plot for the integral uniformity). Looking at the contrast plots, one can say that the more iterations, 


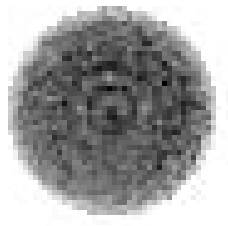

A

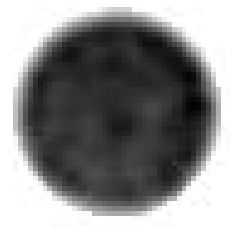

$B$

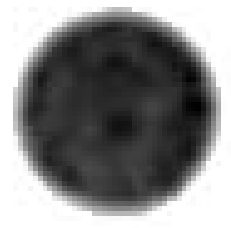

C

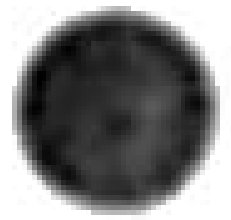

$D$

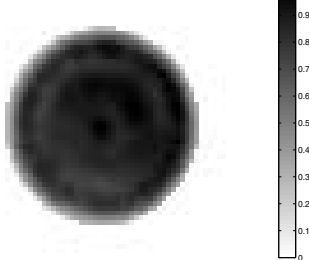

E

Fig. 8. OSEM reconstruction results ( 8 iterations, 4 subsets) for the uniform slice.

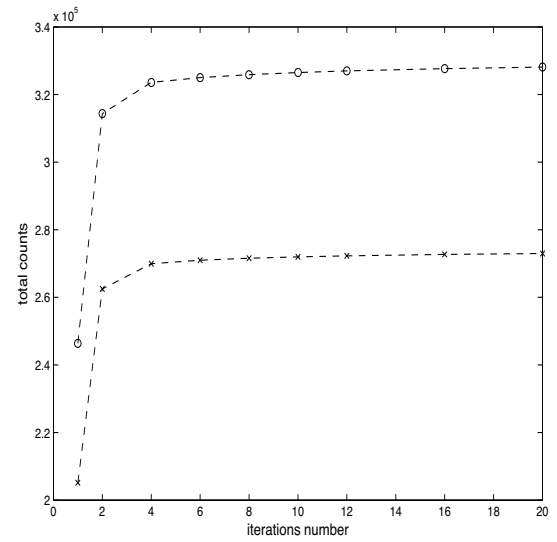

Fig. 12. Total numbers of counts in the reconstructed images: circles represent a slice with "cold" spheres and crosses a uniform slice. We performed 1, 2, 4, 6, 8, 10, 12, 16 and 20 iterations with a 4 subset OSEM algorithm.

the better, but taking into account uniformities and RMS dependence, it can be found that the maximum number of iterations is 16 (for 4 subsets).

At least 6 iterations for 4 subsets are needed to stabilize the total counts in both reconstructed slices (Fig. 12).

\section{Discussion}

In this work, a method of system matrix computation based on direct CDR function measurements and CT-based attenuation correction was verified. It was shown that the CDR function based on a one-dimensional fit provides slightly better results. The two-dimensional surface fit was more difficult and not as good as the one-dimensional case. It was shown by two measurement examples that the Gaussian function in the two-dimensional case is only an approximation. The quality of fit also changes in the onedimensional case- the closer the source is to the detector, the worse the Gaussian fit that can be obtained.

The proposed reconstructions with system matrix computation based on direct measurements produce contrasts and uniformity factors that are in accordance with appropriate ranges proposed by the American Association of Physicists in Medicine (AAPM) in a quality SPECT performance report (Graham et al., 1995). However, because in this study a different isotope of interest was used than the one mentioned in the report (in our case it was ${ }^{131} \mathrm{I}$ instead of ${ }^{99 m} \mathrm{Tc}$, in the report), which entailed different collimators, etc., these ranges cannot be directly compared to our results. However, if the AAPM values are defined for an isotope and collimators with higher spatial resolution, then obtaining those values with ${ }^{131} I$ can be considered an improvement.

Assuming 8 iterations with 4 subsets in our implementation of the OSEM algorithm (which gives 32 iterations for the MLEM algorithm), all the ranges were held, so in our opinion these values are satisfactory.

In this approach, the 3D nature of photon interactions was not taken into account while performing the reconstruction in a slice-by-slice way. Some authors report that fully $3 \mathrm{D}$ reconstruction can improve image quality (Lazaro et al., 2005).

\section{Conclusions}

The method presented for the computation of the system matrix for iterative reconstruction algorithms in emission tomography is simple and much faster than Monte Carlo simulations. It gives the ability to take into account many processes that occur during the imaging procedure. In this case, the measurements are easy and fast to perform, and also simple to repeat. This is very important because it has to be done for every isotope-collimator pair used on a particular machine. A two-dimensional fit provides a similar quality of the resulting images and is required for a fully $3 \mathrm{D}$ reconstruction, which can improve the quality even more. However, a fully 3D reconstruction requires the use of parallel computers or a powerful graphics processor and will be the focus of our future work.

\section{Acknowledgment}

This work was supported by the Polish Ministry of Science and Higher Education under Grant No. N N519 647840. The first version of this paper was presented during the 15th National Conference on the Application of Mathematics to Biology and Medicine (Szczyrk, Poland, 


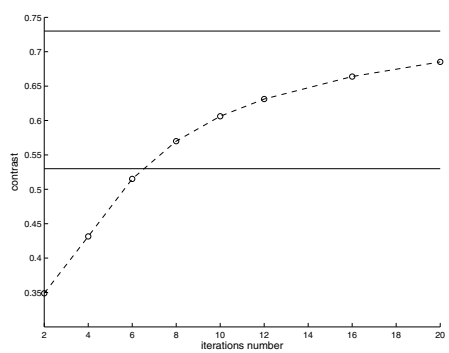

Sphere 1

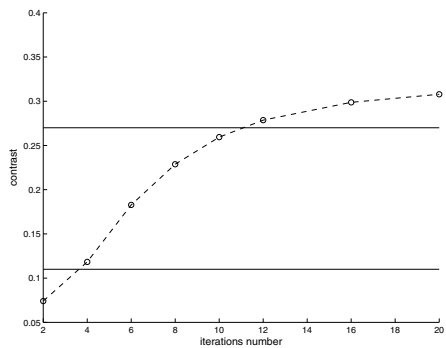

Sphere 4

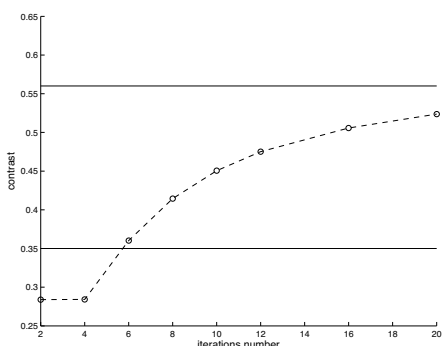

Sphere 2

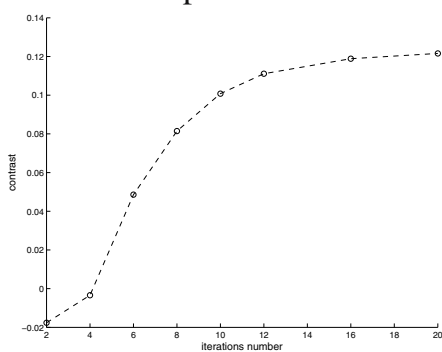

Sphere 5

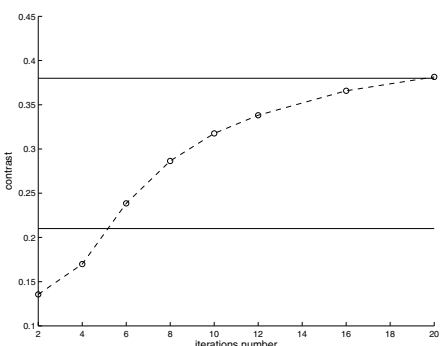

Sphere 3

Fig. 10. Dependence of contrast on the number of iterations. We performed 1, 2, 4, 6, 8, 10, 12, 16 and 20 iterations with a 4 subset OSEM algorithm. The contrast range, if specified, is shown with solid lines.

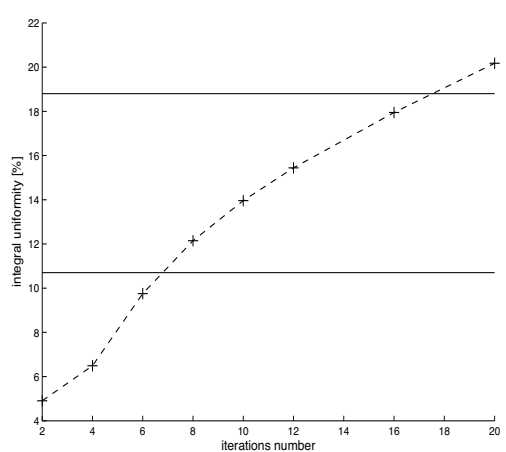

integral uniformity

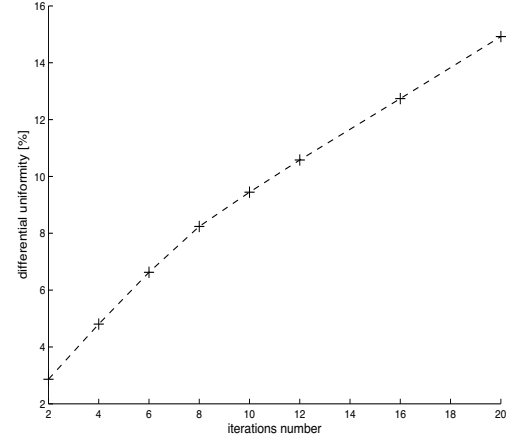

differential uniformity

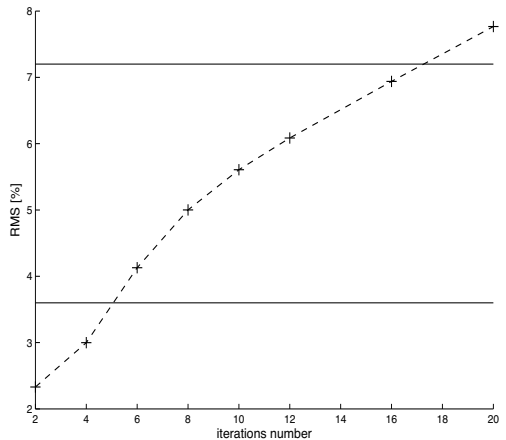

RMS

Fig. 11. Dependence of integral and differential uniformity and RMS on the number of iterations. We performed 1, 2, 4, 6, 8, 10, 12, 16 and 20 iterations with a 4 subset OSEM algorithm. Ranges from the literature, if specified, are indicated with solid lines.

2009) and published in a shortened form in the conference proceedings.

\section{References}

Autret, D., Bitar, A., Ferrer, L., Lisbona, A. and Bardies, M. (2005). Monte Carlo modeling of gamma cameras for I131 imaging in targeted radiotherapy, Cancer Biotherapy and Radiopharmaceuticals 20(1): 77-84.

Borys, D., Panek, R., Gorczewski, K., d'Amico, A., Steinhof, K. and Psiuk-Maksymowicz, K. (2006). Evaluation of SPECT-CT image fusion quality control, Biocybernetics and Biomedical Engineering 26(4): 79-90.

Bruyant, P.P. (2002). Analytic and iterative reconstruction algorithms in SPECT, Journal of Nuclear Medicine 43(10): 1343-1358.
Chang, L.T. (1978). A method for attenuation correction in radionuclide computed tomography, IEEE Transactions on Nuclear Science 25(1): 638-643.

Cherry, S.R., Sorenson, J.A. and Phelps, M.A. (2003). Physics in Nuclear Medicine, Saunders/Elsevier Science, Philadelphia, PA.

Formiconi, A.R., Pupi, A. and Passeri, A. (1989). Compensation of spatial system response in SPECT with conjugate gradient reconstruction technique, Physics in Medicine and Biology 34(1): 69-84.

Gilland, D.R., Jaszczak, R.J., Wang, H., Turkington, T.G., Greer, K.L. and Coleman, R. E. (1994). A 3D model of nonuniform attenuation and detector response for efficient iterative reconstruction in SPECT, Physics in Medicine and Biology 39(3): 547-561. 
Graham, L.S., Fahey, F.H., Madsen, M.T., van Aswegen, A. and Yester, M.V. (1995). Quantitation of SPECT performance: Report of Task Group 4, Nuclear Medicine Committee, Medical Physics 22(4): 401-409.

Hubbell, J.H. and Seltzer, S.M. (n.d.). Tables of X-ray mass attenuation coefficients and mass energy-absorption coefficients, http: / / www.nist.gov/physlab/data /xraycoef/

Hudson, H.M. and Larkin, R.S. (1994). Accelerated image reconstruction using ordered subsets of projection data, IEEE Transactions on Medical Imaging 13(4): 601-609.

Ichihara, T., Ogawa, K., Motomura, N., Kubo, A. and Hashimoto, S. (1993). Compton scatter compensation using the triple-energy window method for single- and dual-isotope SPECT, Journal of Nuclear Medicine 34(12): 2216-2221.

Lazaro, D., El Bitar, Z., Breton, V., Hill, D. and Buvat, I. (2005). Fully 3D Monte Carlo reconstruction in SPECT: A feasibility study, Physics in Medicine and Biology 50(16): 37393754.

Liang, Z., Turkington, T.G., Gilland, D.R., Jaszczak, R.J. and Coleman, R.E. (1992). Simultaneous compensation for attenuation, scatter and detector response for SPECT reconstruction in three dimensions, Physics in Medicine and Biology 37(3): 587-603.

Loudos, G.K. (2008). An efficient analytical calculation of probability matrix in 2D SPECT, Computerized Medical Imaging and Graphics 32(2): 83-94.

Rafecas, M., Böning, G., Pichler, B.J., Lorenz, E., Schwaiger, M. and Ziegler, S.I. (2004). Effect of noise in the probability matrix used for statistical reconstruction of PET data, IEEE Transactions on Nuclear Science 51(1): 149-156.

Shepp, L.A. and Vardi, Y. (1982). Maximum likelihood reconstruction for emission tomography, IEEE Transactions on Medical Imaging 1(2): 113-122.

Vandenberghe, S., D'Asseler, Y., Van de Walle, R., Kauppinen, T., Koole, M., Bouwens, L., Van Laere, K., Lemahieu, I. and Dierckx, R.A. (2001). Iterative reconstruction algorithms in nuclear medicine, Computerized Medical Imaging and Graphics 25(2): 105-111.
Zaidi, H. (2006). Quantitative Analysis in Nuclear Medicine Imaging, Springer, New York, NY.

Zeng, G.L. (2001). Image reconstruction-A tutorial, Computerized Medical Imaging and Graphics 25(2): 97-103.

Damian Borys is an assistant professor at the Institute of Automatic Control of the Silesian University of Technology in Gliwice. He received his M.Sc. degree in computer science and a Ph.D. degree in biocybernetics and biomedical engineering from the Silesian University of Technology in 2004 and 2009, respectively. He is also a member of the Section of Treatment Planning and Molecular Imaging at the Nuclear Medicine and Endocrine Oncology Department. The scope of his research interests includes quantitative medical imaging, multimodal image fusion, medical dosimetry, mathematical modelling, Monte Carlo simulations and parallel computing.

Katarzyna Szczucka-Borys works as a physicist at the Nuclear Medicine and Endocrine Oncology Department of the Comprehensive Cancer Centre and Maria Skłodowska-Curie Memorial Institute and is a member of the Section of Treatment Planning and Molecular Imaging. In 2004 she obtained her M.Sc. degree in medical physics from the University of Silesia in Katowice. Her interests are mainly in the field of calibration and quality control of SPECT, PET and CT systems, medical image processing and registration, and radioisotope labelling.

Kamil Gorczewski is a research assistant at the Nuclear Medicine and Endocrine Oncology Department of the Comprehensive Cancer Centre and Maria SkŞodowska-Curie Memorial Institute. Having obtaining his M.Sc. in medical physics in 2004 , he is currently a Ph.D. student at the Medical Faculty of the University of Tuebingen. The scope of his research includes measurements of water diffusion in human brain, quantitative calibration and quality control of SPECT and PET systems, and multimodal image fusion. He is a member of the Section of Treatment Planning and Molecular Imaging of his home department.

Received: 24 March 2010

Revised: 17 July 2010 